\title{
Motivating Female Students for Computer Science by Means of Robot Workshops
}

\author{
https://doi.org/10.3991/ijep.v10i1.11661 \\ Lisa Keller ${ }^{(凶)}$, Isabel John \\ University of Applied Sciences Würzburg-Schweinfurt, Würzburg, Germany \\ lisa.keller.it@gmx.de
}

\begin{abstract}
As women belong to a minority in computer science, this work aims to provide possible STEM education initiatives for middle to high school students. These initiatives are designed for institutions to contribute to increasing the proportion of women in computer science by using robots. Through applying these initiatives, institutions such as computer science faculties can help to reduce the gender imbalance, increase diversity and the amount of computer science specialists, who are requested immensely. Based on the results of conducted requirements elicitation a concept for programming an application with the NAO robot which trains vocabularies, as well as a concept with the Cozmo robot which piles up cubes, were developed. First tests were performed with female and male high school students. Either concept consists of an introduction of possible robotics application fields, a presentation explaining the Python program code of the concept as well as corresponding exercises with the robot and Python. Evaluation of the workshop concepts through questionnaires showed that both concepts have the potential to increase the proportion of women and to motivate female as well as male students for computer science. Moreover, recommendations for further robotics projects with respect to motivating young women for STEM can be made based on the insights of the requirements elicitation and the evaluation of the concepts. Nevertheless, further refinement and validation has to be undertaken. Long-term as well as short-term initiatives are feasible with the developed concepts. All participating schools showed high interest in both types of initiatives and further cooperation.
\end{abstract}

Keywords-Women in computer science, proportion of women in STEM, diversity, robotics in education.

\section{Introduction}

Women belong to a minority in computer science (CS). It is said that women bring a different mindset and therefore are highly needed in CS [1]. However, women seem to see STEM and CS as a male topic [2]. But how can we achieve to motivate young women to gain interest in CS or related STEM subjects?

Robots are used with pleasure in education as they can be perceived as friends by students [3]. Moreover, students have little experiences with robots. Due to novel and 
practical experiences, robots can raise curiosity, interest, and motivation in students for STEM subjects [3, 4].

Other studies have shown that robotics concepts have potential to increase the proportion of interested female students $[5,6,7]$. The results of the project Roberta-EU, which used Lego Mindstorms robots, show that many female participants became interested in STEM [5]. Also, the results of another study, which participated with girls at Botball, demonstrate that robotics project can have a positive impact on girls' attitude towards STEM [6]. Moreover, a related work offering STEM courses at high schools, observed higher interest of women in information systems bachelor courses [7].

Based on this perception, this work initially developed in a bachelor thesis [8, 9], aims to develop new workshop concepts, especially designed to motivate young women for CS by means of robots. These concepts are applied as workshops, which can be used by CS faculties to contribute to increasing the proportion of female CS professionals.

Moreover, this work is also targeting to improve the success rate of women robotics projects by analyzing the needs of the target group through requirements elicitation, which was not undertaken by previous studies in this form. Through the newly gained insights of the target group's needs, robotics projects can be further enhanced to increase the probability of motivating young women for CS even more.

\section{Methods}

To gain insights how robots should look like and act in order to appeal to the target group, a mixed methods study, was performed with female students aged 12 to 16 by means of Requirements Engineering (RE) with Lego, brainwriting, a workshop, and a questionnaire. The study was performed as a workshop with seventeen participants during a half-day "Girls Day" event (https://www.girls-day.de/) at the authors' faculty. Based on these results two new workshop concepts were developed and tested with female as well as male high school students aged 15 to 20. These two-hour workshops were performed with interested high school classes and working groups at their schools. Finally, the concepts were evaluated through questionnaires. All RE methods were chosen with reference to a RE guideline [10]. As the goal was to find innovative requirements, the initial elicitation was designed fairly open to give the participants the chance to bring in new ideas. Therefore, open and indirect techniques such as Lego robot building and brainwriting were chosen.

\section{Requirements Elicitation}

In order to gain insights on how a robot should appear to appeal to the target group, seventeen female participants (aged 12 to 16) built robots with Lego, how they think a robot should look like. Moreover, the behaviors a robot should be capable of according to the target group were determined through brainwriting. In addition, a presentation containing short videos on possible robotics application areas was shown and a work- 
shop with the humanoid robot NAO (see Figure 6) was performed. At the NAO workshop, the girls could gain their first hands-on experience and program the robot with its visual programming language. Finally, favorite robotics application fields, additional robotic appearance and behaviors as well as further interest were identified through a questionnaire [10].

\subsection{Results}

Built lego robots: Three two-legged as well as three four-legged Lego robots were built, as seen in Figure 1. Moreover, three of the robots have two arms.
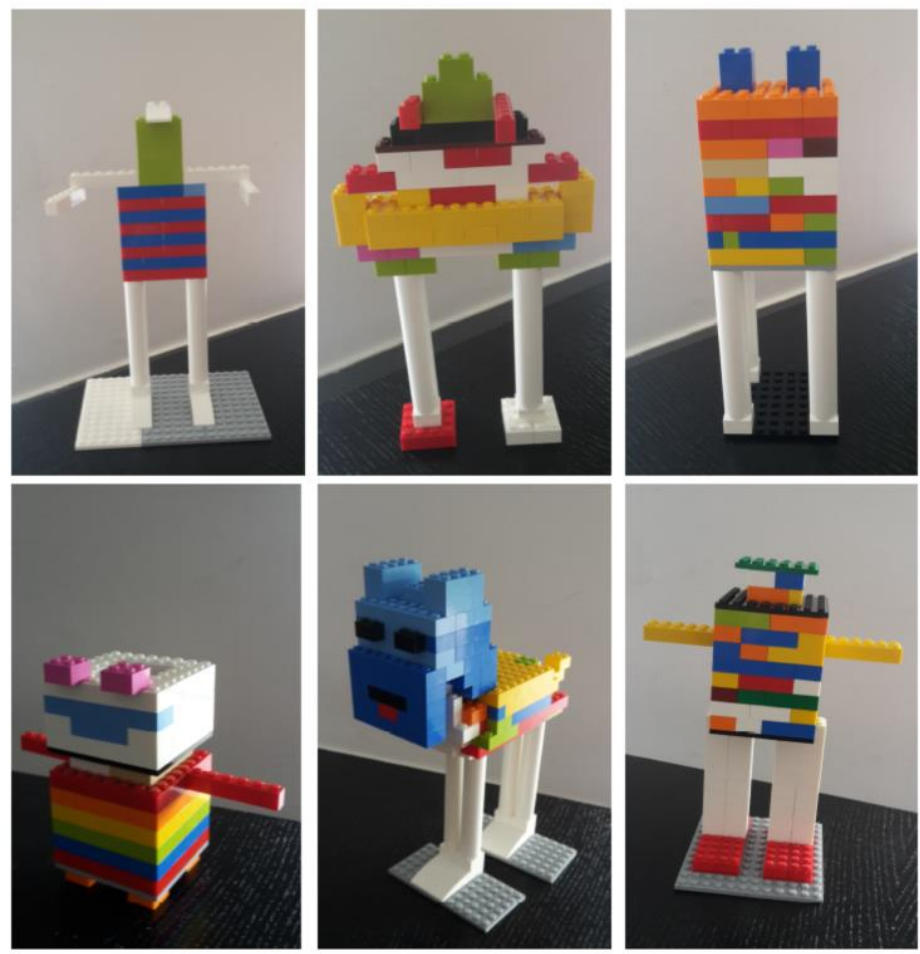

Fig. 1. Built Lego robots

Brainwriting: The resulting behaviors a robot should be capable of were clustered into different categories:

- Daily Life: Cleaning, cooking, housekeeping, grocery shopping, arranging appointments, butler, planning holidays, googling.

- Entertainment: Dancing, singing, playing music, playing the guitar, doing sports (football, hockey, gymnastics, riding, swimming, diving), drawing, magic tricks, entertaining, painting fingernails. 
- Languages: Reading, writing, speaking, having a conversation, translating, being capable of all languages.

- Social, Work, and Help: Helping people, doing homework, explaining words, calculating, doing maths, teaching, helping with homework, having a job, repairing.

- Human or Animal-Like: Should have emotions, should be like a dog, should have its own opinion and character, be like a friend, laughing, running, should react if called, should do everything it is told, teach commands to the robot, follow a person, movements (walking, waving, moving objects, throwing, fighting).

Questionnaire: Based on the presentation introducing possible robotics application fields, the participants filled in a questionnaire [10]. The presentation included short videos of the following application areas and corresponding robots: healthcare (exoskeletons), education (Lego Mindstorms), entertainment (Furby robot), social interaction (Pepper robot), research (mars rover), industry (Baxter robot), and military (EOD robot). As shown in Figure 2, the participants ranked the application fields they are most interested in as follows: healthcare (82\%), social interaction (82\%), entertainment $(77 \%)$, research $(70 \%)$, education $(70 \%)$, military $(47 \%)$, and industry $(36 \%)$.

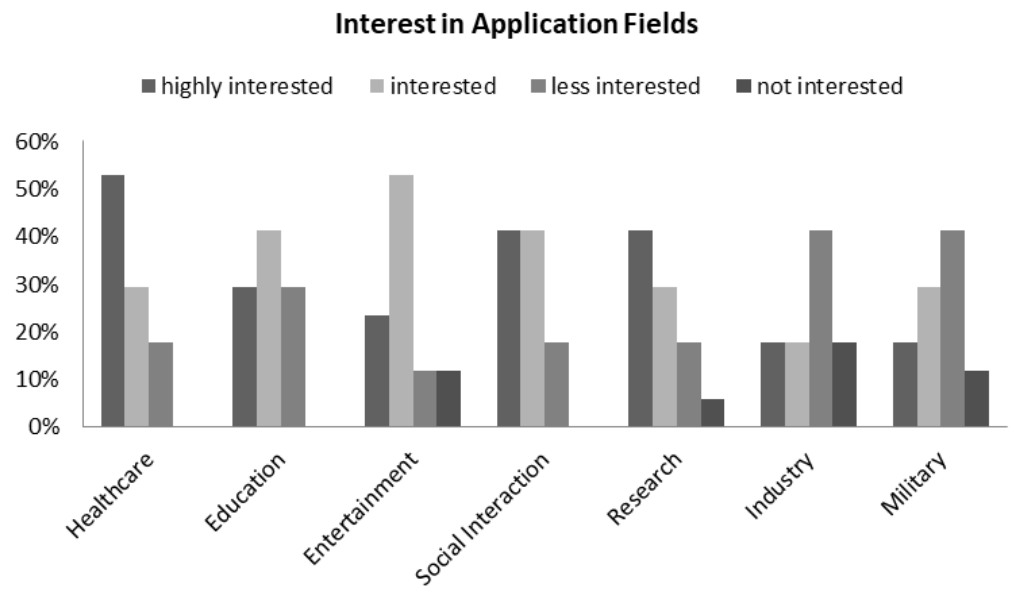

Fig. 2. Interest in application fields

The percentage share of the ranking was determined through adding the values of highly interested and interested. If more application fields have the same value, the application field with higher percentage of highly interested was ranked first. Moreover, the target group specified that they were most fascinated by Pepper (social interaction) and the exoskeleton (healthcare) because they can be useful and help people.

Another question addressed how humanoid a robot should be to appeal to the target group. Therefore, several pictures of robots which become more humanoid from left (TurtleBot) to right (Android Erica) were arranged in the questionnaire, see Figure 3. The participants would like to occupy most with Aldeberan's NAO (g), Fraunhofer 
IPA's Care-O-bot (e), Hiroshi Ishiguro's android Erica (i), and Lego Mindstorms NXT's puppy (c).

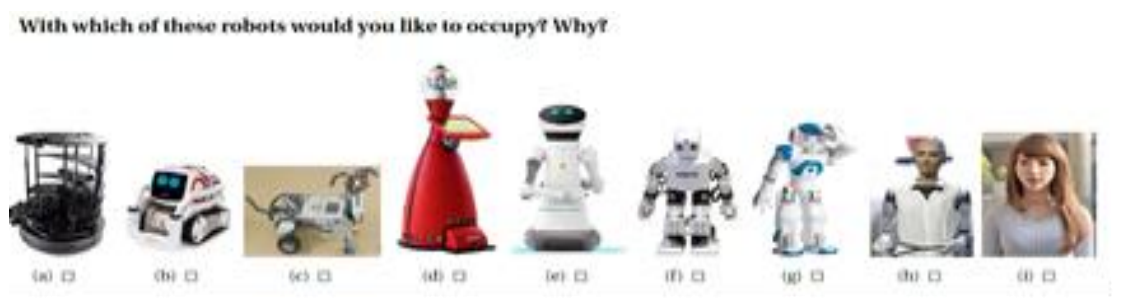

Fig. 3. Question on different robot types

The reasons stated were that (g) looks cute, humanoid but not too much, and interesting. Robot (e) was chosen due to its cute looking and butler similar appearance. Erica (i) was selected because it looks like a human and the wish to talk to it arose. Finally, (c) was preferred due to cuteness and dog similar appearance. Anki's Cozmo (b) and Robotis Darwin-Mini (f) were favoured moderately.

On the other hand, the robots the participants would like to occupy with least are Turtle-Bot $2 \mathrm{i}$ (a), MetraLabs SCITOS A5 (d), and Hanson Robotics' mechanical looking android Sophia (h). An interpretation could be that, although (h) looks humanoid, it still looks like a robot, having no hair and is missing the cuteness the other favored robots have.

Also, the participants' wishes for next girls' days were asked to see if we matched the interest of the target group and to also identify further potential to gain young women's interest in robots.

The results (see Figure 4) show that the interest of the target group was already met fairly well. The interest in more sophisticated topics such as exoskeleton or more interaction/speaking was fairly small, so the workshops should be generally kept in this form.

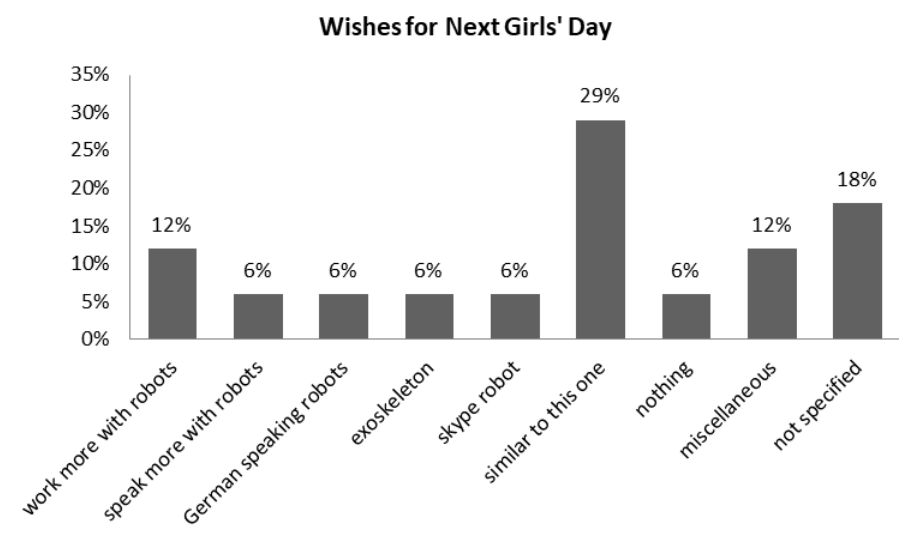

Fig. 4. Wishes for further topics 
Finally, to verify if interest in STEM arose, the participants were asked if they are interested in further offers. They could also specify their email address if they liked. As Figure 5 indicates, $70 \%$ of all participants are interested in further offers regarding robotics workshops and roughly $40 \%$ provided her email address.

\section{Interest in Further Offers}

$\square$ Yes $\square$ Yes and email address $\square$ No $\square$ Miscellaneous

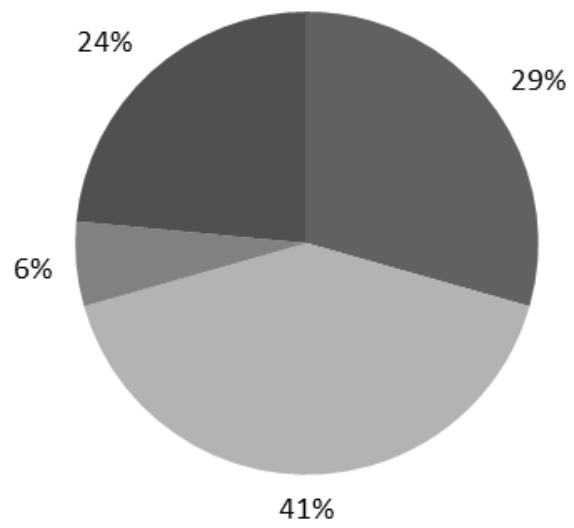

Fig. 5. Interest in further offers

\subsection{Analysis}

The built Lego robots seen in Figure 1 indicate that humanoid and animal-like robots show potential to motivate young women for CS as all build Lego robots exhibit human or animal traits. Also, the human or animal-like cluster of brainwriting emphasizes this. Thereby, these insights correspond to the uncanny valley hypothesis [11, 12]. However, the android Erica which was highly favored by the target group would fall into the uncanny valley. Therefore, androids or programmable dolls could also show promise to motivate young women for STEM, even though they might fall into the uncanny valley. However, further research has to be undertaken in this area.

Nevertheless, the results of RE imply that robots do not have to appear humanoid or animal-like necessarily in order to motivate girls for STEM, as the participants were also highly fascinated by exoskeletons. However, non-humanoid or animal-like robots should also belong to the interested application fields and behaviors determined at RE.

By combining all insights, the robots which can cause higher potential to motivate girls for STEM can be classified as terrestrial, aquatic, or aquatic-terrestrial service robots [13] which operate in healthcare, social interaction, entertainment, research, and education. 
Interestingly, cuteness and humaneness seem to be equally appealing to the target group, as the question on robot types shows. So, building or using a robot that is interesting for female students should either look like a cute animal, a toy or puppet, or it should resemble a likeable person.

Too technical or serious looking robots seem to be too frightening to the target group. Especially, the low rating of the mechanical looking android Sophia (h) in Figure 3 could be an interpretation that the function/purpose of the robot has to be clear. Sophia does not really look like a robot but also not exactly like a human. This might be slightly frightening to the target group.

Considering the wishes for further topics in Figure 4 it can be concluded that the expectations of the target group with respect to the workshops were met. Therefore, we can continue to offer the workshops as they currently are. Nevertheless, there is always room for improvement. So, in the meantime we experimented with modified workshops with Cozmo robots with younger student groups (aged 11-13). Therein, we concentrated on playing and interacting with the robots and supported self-efficacy of the girls in interacting with the robots by giving them small and easy programming tasks like personal salutation by the Cozmo robot.

Finally, the results illustrated in Figure 5 show that nearly three-quarters of all participants are interested in further robotics workshops and roughly $40 \%$ provided their email address, which emphasizes high interest. This indicates that measures with respect to motivating young women for STEM can have a positive impact.

\subsection{Threats to Validity}

The conducted study was a mixed method study but because of the relatively low number of participants the focus was on qualitative statements [14] and surely did not produce resilient results. The study was rather used for requirements elicitation and generating further hypotheses than for producing countable results. The group of students that was participating at the workshop all came from the area of Würzburg. So, there might be a regional bias. Perhaps, results from other regions in Germany or other countries might look different due to other cultural backgrounds. The students participated voluntarily at the workshops. So, they already might have a general interest in STEM, computer science or even in robots. It would be interesting to replicate the study with a random student group of same age and a different cultural background.

\section{New Workshop Concepts}

Based on the insights of RE, two new workshop concepts with the robots seen in Figure 6 were developed. The NAO robot [15] trains vocabularies with the students through speech recognition, whereas the Cozmo robot [16] talks, piles up cubes, and sings. Thereby, the concepts suit to the categories: entertainment, languages, human or animal-like as well as social, work, and help (see section 3). Moreover, the workshops belong to the application fields of social interaction, entertainment, and education 
which are favored by the target group. Likewise, the NAO and Cozmo robot were chosen due to their humanoid and animal-like appearance, respectively.

Either workshop consists of an introduction of possible robotics application fields, a presentation explaining the Python program code of the concept as well as corresponding exercises with the robot and Python. At the vocabulary training workshop, the students had to adjust the IP of the robot, add five words to the already existing vocabulary trainer, and to let the robot introduce itself verbally. On the other hand, at the pile up cubes workshop, the students had to change the notes of the song and the speed of driving, let the robot introduce itself, and finally let it drive in a rectangle.
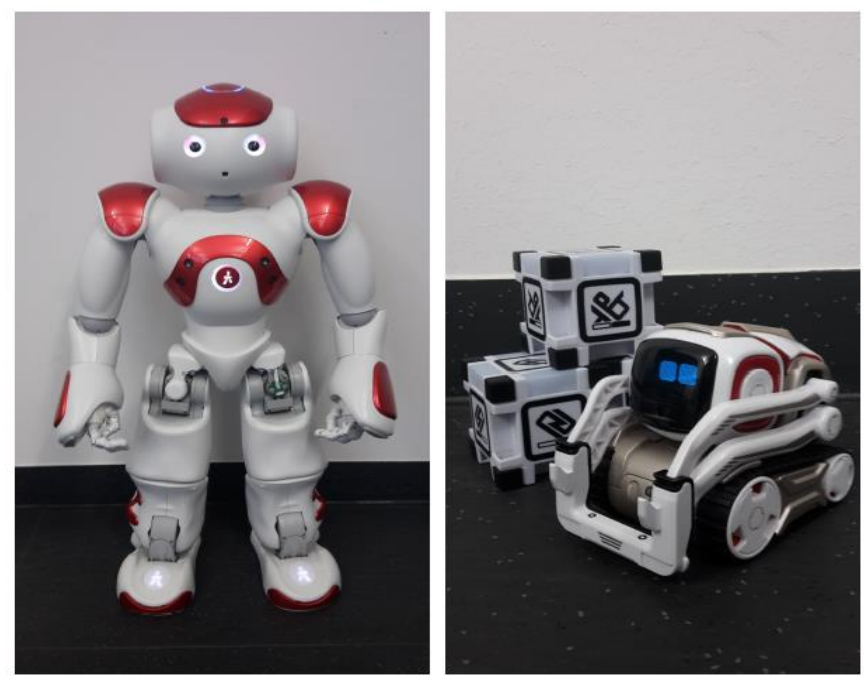

Fig. 6. Aldebaran Robotics' NAO (left) and Anki's Cozmo (right)

\section{Evaluation of New Workshop Concepts}

The vocabulary workshop concept was tested with nine female and eight male high school students aged 17 to 20 in grade 12 . Likewise, the pile up cubes concepts was tested with fourteen female and nine male students aged 15 to 18 in grade 10. Both concepts were evaluated by means of a questionnaire $[10,14]$. To see if the workshops cause positive changes in the thoughts of the students, they were asked what they thought about CS before and after the workshop. Moreover, the students' feelings during the workshops and exercises as well as their perceived difficulty were gathered. Furthermore, their favorite robot behaviors and highlight of the workshop as well as wishes for another workshop were asked. In Addition, the students' further interest in STEM could be identified through the questionnaire. The results and analysis are outlined in the following. 


\subsection{Results}

Vocabulary training: Before the vocabulary training workshop, about one-fifth of the female and one-quarter of the male students thought that CS is interesting. After the workshop, over half of the female and one-quarter of the male were more interested in CS. Only the thoughts of approximately one-fifth females and one-quarter males remained unchanged.

All participants' feelings during the workshop were nearly entirely positive. Only roughly one-tenth of the male felt unwise. During the exercise half of the female as well as half of the male specified positive feelings, whereas about one-tenth of the female stated that they were overstrained.

The participants were asked to rank how they perceived the difficulty of the workshop and the exercises on a scale from 1 to 6 , whereas 1 means simple and 6 difficult. The perceived difficulty of the workshop is similar but differently distributed comparing both sexes. Both ranked the difficulty of the workshop from 2 to 4 , whereas scale 3 was selected most. The exercises were perceived as more difficult by both sexes and were ranked as 4 mostly.

The most favorite robot behavior of female as well as male students is dancing, followed by the developed concept of vocabulary training. Also, playing and communicating with humans were favored by females as well as push-ups which were also favored by male students. The reasons why they liked these behaviors most were that they did not think a robot could dance as well as that it appeared human. Moreover, they enjoyed training vocabularies with the robots because it is useful and they also thought that only humans can train vocabularies. In addition, push-ups were favored because it is funny and entertaining.

The most mentioned highlight of the concept was the NAO robot itself, according to the female students. Moreover, that they saw the possibilities of robots, learned something about computer science, and saw a robot dancing were further highlights. At the same time, the most mentioned highlights of the male students were to see something new and the possibilities of robots. Furthermore, they enjoyed the NAO robot itself as well as the robot dancing, doing push-ups and headbanging to ACDC's song "Highway to Hell".

For further workshops, almost $40 \%$ of the female would wish for nothing, nearly $30 \%$ for other robot behaviors, and roughly $20 \%$ for more programming exercises. At the same time roughly one-tenth of the male participants would like to have bigger robots, other robot behaviors, and autonomous driving, in each case.

Moreover, all participants would recommend the workshop because it was enjoying, exciting and interesting to see what can be done with CS, as stated by some female participants. Finally, the students were asked if they would participate in a robotics group if their school would offer one. One-third of the female and one-quarter of the male students would participate in the group as indicated in Figure 7. The girls would participate because it is interesting to work with robots and to see the results. Moreover, the boys said that robotics is useful and highly interesting. On the other hand, the girls who would not like to participate stated that they had no time for it, are not good in CS, think they will not understand it, or cannot identify with it. Furthermore, the boys who 
would not like to participate said that they are not interested in CS or are too less technical skilled.
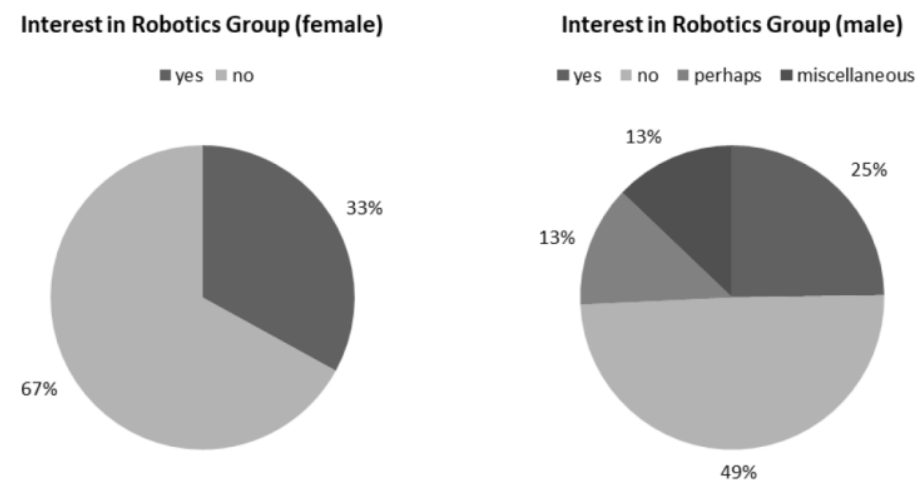

Fig. 7. Vocabulary training: Interest in robotics group

Pile up cubes: Approximately $5 \%$ of the female and $40 \%$ of the male participants had positive thoughts about CS before the pile up cubes workshop. Afterwards however, over $60 \%$ of the females and over $40 \%$ of the males thought positively about CS. The thoughts of approximately one-fifth females and nearly the half of males remained unchanged.

The female participants' feelings during the workshop were nearly entirely positive. Also, during the exercises most declared favorable feelings. However, nearly one-tenth felt unsure, overstrained, or that the exercise was difficult, respectively. On the other hand, the male students also stated mostly positive feelings during the workshop and exercises.

The difficulty of the workshop was ranked on scale 2 and 3 by the female students, while scale 3 was ranked most by almost $60 \%$. At the same time the male students ranked from 1 to 3 , whereby scale 3 was ranked most by nearly half and scale 2 second most by one-third. On the other hand, the difficulty of the exercise was rated from scale 2 to 4 . Thereby, scale 2 was most ranked by the female students by nearly $40 \%$.

The most favorite robot behavior of female as well as male students is speaking because it is funny according to the participants. The female favored playing second most followed by object and face recognition as well as singing third and fourth most, respectively. On the other hand, the male students enjoyed playing with the robot, flashing lights of the cubes, emotions of the robot as it is surprising, as well as object and face recognition second most.

The greatest highlights of the lesson for the female students were that they could try and work with the robots themselves as one-third stated and the Cozmo robot itself which was declared by nearly $30 \%$. Other highlights were playing with Cozmo, and that Cozmo could speak. On the contrary, that Cozmo could speak was the greatest highlight of male students as nearly half of them stated. Furthermore, they also specified to try the robot themselves, play with Cozmo, programming, everything, and the tasks as highlights. 
For further workshops, half of the female students wished to spend more time with the robot. Nearly $15 \%$ stated that they still like to work with the robot themselves and almost one-tenth wished to do more complex and different tasks. On the other hand, the male students wished to build own robots, work more with the robot themselves, to have more groups, and to speak more with the robot.

Moreover, all participants would recommend the workshop because it was interesting, practical, and enjoying according to the participants. As the school where this workshop was tested offered a robotics group, the students were asked if they currently participate in and if not, if they would participate after this workshop. As Figure 8 shows no one of the female students did currently participate and only $14 \%$ would participate perhaps. One female participant stated that she is interested and thought about participating in the robotics group but would not because she said that she is not technically gifted. Other females stated that CS is nothing for them or that they are not very interested in CS. Also, none of the male students did currently participate in the robotics group. However, around one-tenth had participated previously and quitted due to stress. Almost a quarter would participate in the group and approximately one-tenth perhaps.

Interest in Robotics Group (female)

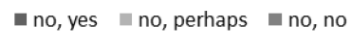

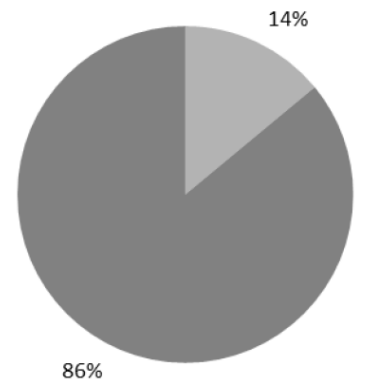

Interest in Robotics Group (male)

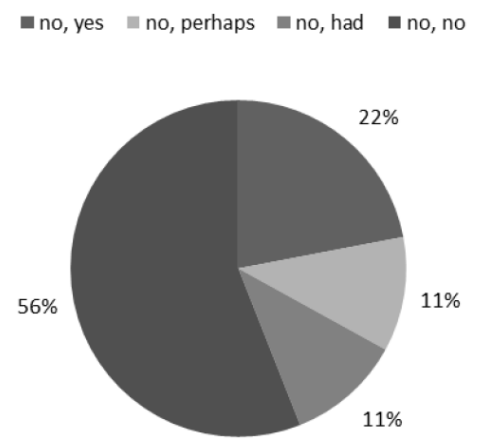

Fig. 8. Pile up cubes: Interest in robotics group. The first yes or no refers to current participation in the robotics group and the second if they would participate.

\subsection{Analysis}

Both workshops demonstrate the potential to motivate students for CS, as most of the female and male participants thought more positively of CS and were more interested in it after the workshops. However, the workshops seem to have a higher impact on chancing the females' thoughts of CS to the better, as more female students specified positive thoughts after both workshops than male students.

Having a favorable atmosphere is a crucial factor for motivating people. As all students almost entirely stated positive feelings during both workshops and exercises, the basic prerequisite for motivating students is met. 
Both workshops are suitable to use for students without prior programming knowledge, as the perceived difficulty was rated moderately. The feeling of being overstrained some female participants faced at both exercises, can be reduced by additional support.

As inter alia the vocabulary training concept itself and dancing were perceived as surprising, entertaining and useful, they were the students' most favorite robot behaviors. This implies that workshop concepts show high potential in motivating female as well as male students for CS if they are perceived as such.

Moreover, it can be implied that the NAO robot was well-chosen for the concept as it was the highlight of many female students. However, less male students stated the NAO robot as highlight. This might imply that the appearance of robots is less important to men than to women.

As most female students wished for nothing more at the vocabulary concept, they seemed to be satisfied. This supports the eligibility of the concept.

The pile up cubes concept contains the most favorite behaviors: speaking, singing as well as object and face recognition. This aspect also supports that this concept shows potential to increase the proportion of women in CS and that the concept is also applicable for men. Moreover, the results of the favored behaviors sustain the results of requirements elicitation (see section 3 ).

The Cozmo robot seems to be well-chosen for the concept because the Cozmo robot itself, speaking, and playing with it were the highlights of both sexes.

As half of the female students wished to spend more time with the Cozmo robot at further workshops, this implies that they enjoyed the time with the robot. Furthermore, it suggests that the time of the concept should either be extended or the presentation shortened in order to enable the students to spend more time with the Cozmo robots.

Furthermore, all participants would recommend the workshops, which can indicate further interest and more positive thoughts about CS, respectively. This is only an indirect measure for motivation, but a more direct measuring like counting the number of enrollments in CS some years later is not possible yet.

The results illustrated in Figure 7 show that more female students $(33 \%)$ would participate in a robotics group than their male $(25 \%)$ fellow students. This shows, that the vocabulary training workshop shows potential to motivate young women for CS. Thereby, CS faculties can contribute to increasing the proportion of women in CS by applying the workshop. However, the results demonstrate that also male students can be motivated with the workshop.

On the other hand, more male students would participate in a robotics group than female students with respect to the pile up cubes workshop as the results in Figure 8 indicate. Although, the results shown in Figure 8 do not turn out highly promising to increasing the proportion of women in CS, the pile up cubes workshop still shows potential to gain impact and motivate women for CS, as the thoughts of CS of over half of the female students changed for the better. Albeit, the vocabulary training workshop shows higher potential to motivate women for CS than the pile up cubes workshop does. 


\subsection{Threats to Validity}

During the pile up cubes workshop, technical difficulties occurred as some laptops did not recognize the linked android device which is needed to program Cozmo through the Python SDK. Thus, some students had less time to do the exercise as they could only do it after the issues were fixed. These technical difficulties could have caused that less female students would like to participate in a robotics group. Moreover, this could also explain why the level of difficulty of the pile up cubes workshop was ranked relatively low, especially compared to the vocabulary training workshop's ranked exercise difficulty level.

Asking about the interest of students for further workshops in an indirect measure for motivation but it does not really quantify the motivation for CS. There is just the hope that the positive image that the female students received from the workshops helps in influencing their decision for CS or a STEM related topic in the future.

\section{Conclusion and Outlook}

Both concepts developed in this STEM education initiative can be recommended to motivate female as well as male students for CS and robotics. Furthermore, the workshop concepts also show promise to increase the proportion of interested women, as barriers of starting to occupy with CS can be decreased. However, the vocabulary training concept shows higher potential to motivate young women for CS. Nevertheless, further refinement and validation has to be undertaken in order to gain higher data accuracy.

Random samples of previous related workshops have shown that they influenced young women's decision of studying CS. However, this effect has not been systematically evaluated and quantified yet. Further work will include the observation of midterm and long-term effects on the students by undertaking follow-up evaluations of the workshops' participants and also evaluations at CS studies enrollment.

Moreover, the insights of the RE and the evaluation of the concepts can be recommended as guideline for further robotics projects and workshops targeting to motivate young women for CS.

Long-term as well as short-term initiatives are feasible with the developed workshops. All participating schools showed high interest in both types of initiatives and further cooperation.

\section{$7 \quad$ References}

[1] D. R. Peiris, P. Gregor and I. V, "Women and computing breaking the cycle of imbalance at the interface", in Women, Work and Computerization Charting a Course to the Future, E. Balka and R. Smith, Eds., Springer, 2000, pp. 34-41. https://doi.org/10.1007/978-0-38735509-2_5

[2] J. Rodriguez, I. Esparragoza "Motivation of Engineering Students Participating in Multinational Design Projects", IJEP Vol 7, No 4 (2017) 
[3] D. P. Miller and I. Nourbakhsh, "Robotics for education", in Handbook of Robotics, B. Siciliano and O. Khatib, Eds., Springer, 2016, pp. 2115-2134. https://doi.org/10.1007/978-3$\underline{319-32552-1 \_79}$

[4] E. Demertzi, N. Voukelatos, Y. Papagerasimou, A. S. Drigas, "Online Learning Facilities to Support Coding and Robotics Courses for Youth", IJEP Vol 8, No 3 (2018). https://doi. org/10.3991/ijep.v8i3.8044

[5] Fraunhofer Society, "Roberta-eu final activity report", CORDIS Community Research and Development Information Service, Sep. 2009.

[6] J. B. Weinberg, J. C. Pettibone, S. L. Thomas, M. L. Stephen and C. Stein, "The impact of robot projects on girls"e attitudes toward science and engineering", Proceedings of The 5th Annual National Conference on Educational Robotics, Jun. 2007.

[7] C. B. Santos, D. J. Ferreira, A. R. Martins and M. C. B. do Nascimento Rodrigues de Souza, "Robotics and programming: Attracting girls to technology", 2016 International Conference on Advances in Computing, Communications and Informatics (ICACCI), Jaipur, pp. 20522056, Sep. 2016. https://doi.org/10.1109/icacci.2016.7732353

[8] L. Keller and I. John, "How Can Computer Science Faculties Increase the Proportion of Women in Computer Science by Using Robots?" in 2019 IEEE Global Engineering Education Conference (EDUCON), Dubai, 2019, pp. 213-217. https://doi.org/10.1109/ educon.2019.8725212

[9] L. Keller, "How Can Computer Science Faculties Increase the Proportion of Women in Computer Science by Using Robots?", Research Gate Project. [Online]. Available: https://www.researchgate.net/project/How-Can-Computer-Science-Faculties-Increase-theProportion-of-Women-in-Computer-Scienceby-Using-Robots/. https://doi.org/10.1109/edu con.2019.8725212

[10] K. Pohl and C. Rupp, Requirements Engineering Fundamentals: A Study Guide for the Certified Professional for Requirements Engineering Exam - Foundation Level - IREB Compliant, 1st, Rocky Nook, 2011, pp. 24-31.

[11] M. Mori, „The uncanny valley ${ }^{e e}$, IEEE Robotics \& Automation Magazine, vol. 19 Issue 2 June, pp. 98-100, 2012

[12] A. P. Saygin, T. Chaminade, H. Ishiguro, J.Driver and C. Frith, "The thing that should not be: Predictive coding and the uncanny valley in perceiving human and humanoid robot actions", Social Cognitive and Affective Neuroscience, Oxford University Press, vol. 7, pp. 413-422, Apr. 2011. https://doi.org/10.1093/scan/nsr025

[13] M. Ben-Ari and F.Mondada, Elements of Robotics, 1st. Springer, 2018, pp. 1-9.

[14] C. Woehlin, P. Runeson et.al. Experimentation in Software Engineering, Springer, 2012.

[15] Aldebaran Robotics. NAO developer guide [Online]. Available: http://doc.aldebaran.com/24/family/index.html

[16] Anki. Cozmo developer [Online]. Available: https://developer.anki.com/

\section{Authors}

Lisa Keller is a researcher at the University of Applied Sciences WürzburgSchweinfurt (FHWS) in Würzburg, Germany. Moreover, she taught basics of computer engineering, computer architecture and basics of theoretical computer science as student teaching assistant. She is interested inter alia in engineering education, women in STEM, robotics, international projects, human-computer and human-robot interaction. 
Isabel John is a professor for Software Engineering at the University of Applied Sciences in Würzburg, Germany (FHWS). As computer science study program leader and women's representative in the faculty of computer science and business information systems, she has a strong interest in motivating young women to studying computer science and being a software engineer. She does research in the field of gamification and women in CS. Moreover, she teaches software engineering, software architecture, algorithms and data structures as well as other subjects.

Article submitted 2019-09-12. Resubmitted 2019-11-05. Final acceptance 2019-11-05. Final version published as submitted by the authors. 